\title{
Thermodynamic aspects of nonequilibrium current fluctuations
}

\author{
D. Jou, J. E. Llebot, and J. Casas-Vázquez \\ Departament de Termologia, Facultat de Ciències, Universitat Autònoma de Barcelona, \\ Bellaterra, Barcelona, Spain
}

(Received 21 October 1981)

\begin{abstract}
Starting from a macroscopic nonequilibrium entropy, we obtain an expression for the nonequilibrium fluctuations of the electric current in a metallic resistor. Our method goes further than previous theories of irreversible thermodynamics and, as well as microscopic entropies, it leads to results of the same order of magnitude but not completely coincident with the full nonequilibrium corrections obtained from kinetic methods by Tremblay et al.
\end{abstract}

\section{INTRODUCTION}

In the last years, great attention has been devoted to the problem of nonequilibrium fluctuations of thermodynamic systems, ${ }^{1-3}$ since this analysis is directly related to experiments (for instance, light scattering, neutron scattering, computer simulation) and since it allows a deeper insight into the problems of nonequilibrium statistical mechanics. Recently, we have analyzed the problem of equilibrium fluctuations of dissipative fluxes in the framework of the so-called extended irreversible thermodynamics, ${ }^{4,5}$ where, starting from a generalized Gibbs equation and the Einstein hypothesis for the probability of the fluctuations, we have obtained expressions for the second moments of the fluctuations of heat flux, electric current, ${ }^{6}$ and the hydrodynamic dissipative fluxes, ${ }^{7}$ in equilibrium. On the one hand, our theory unifies the usual expressions of the fluctuation-dissipation formulas for the fluctuations of the dissipative fluxes. On the other hand, it places some restrictions on the possible nonequilibrium generalizations of the Gibbs equation, which had not been taken into account up to now.

Recently, we have extended our method to the analysis of nonequilibrium fluctuations of the heat flux in some rigid heat conductors, and we have evaluated the nonequilibrium corrections to the classical Landau-Lifshitz formulas for dielectric solids and metallic conductors. ${ }^{8}$ The purpose of this paper is to apply our method to obtain some information in the influence of an external electric field on the current fluctuations, and to compare our results with the expressions calculated by Tremblay et $a l .^{9,10}$ by a nonequilibrium diagram- matic perturbation theory, and by kinetic arguments.

\section{NONEQUILIBRIUM GIBBS EQUATION}

Extended irreversible thermodynamics is, essentially, a mesoscopic description of thermodynamic systems. While in the usual macroscopic theory the state of a rigid electrical conductor is described by $u$, the specific internal energy per unit mass, and $c_{e}$, the electron density, in the formalism of extended irreversible thermodynamics one takes as supplementary, independent variables the dissipative fluxes, in order to obtain a more detailed description than the usual one. In the present problem, we take as an independent variable the electric current $\overrightarrow{\mathbf{J}}$. In order to achieve a maximum simplicity, we assume that the electron density $c_{e}$ is uniform and constant, and we do not consider the effects of the heat flux. In this paper we do not give a complete description of the method used by the extended irreversible thermodynamics, which has been given already in the literature. , $^{4}$

The evolution equation for the internal energy $u$ is given by the well-known balance equation

$$
\rho \dot{u}=\overrightarrow{\mathrm{J}} \cdot \overrightarrow{\mathrm{E}},
$$

which expresses that the time derivative of the internal energy is equal to the Joule heating. In order to obtain an evolution equation for $\vec{J}$ one can start from a microscopic model (a Boltzmann equation for the electrons, for instance) or one can proceed in a macroscopic way starting from phenomenological hypotheses. We take this latter point of view and try to obtain an evolution equation for $\overrightarrow{\mathrm{J}}$ by following a procedure parallel to that of irreversible thermodynamics. ${ }^{11}$ The difference 
with the latter formalism is that, in our case, $\vec{J}$ is included as a variable in the generalized nonequilibrium entropy in order to obtain some information about its evolution equation from a suitable formulation of the second law. If one assumes that the nonequilibrium entropy $s$ is differentiable enough, the corresponding generalized Gibbs equation is given by ${ }^{6}$

$$
d s=\Theta^{-1} d u-\alpha \overrightarrow{\mathbf{J}} \cdot d \overrightarrow{\mathbf{J}},
$$

where $\alpha$ can be shown to be $e^{6,7}$

$$
\alpha=\left(\frac{\tau v}{\sigma T}\right)
$$

and $\theta$ is a generalized nonequilibrium absolute temperature given by the following equation of state $^{5}$ :

$$
\Theta^{-1}=T^{-1}-\frac{1}{2}\left(\frac{\partial \alpha}{\partial u}\right) \overrightarrow{\mathbf{J}}^{2} .
$$

In these expressions, $T$ is the local equilibrium absolute temperature, $\sigma$ the electrical conductivity, $\tau$ the relaxation time of the electric current, and $v$ the specific volume per unit mass. The equation of state (3) may be obtained ${ }^{6}$ by comparing the constitutive equation for the electric current

$$
\frac{\partial \overrightarrow{\mathrm{J}}}{\partial t}=-\tau^{-1}(\overrightarrow{\mathrm{J}}-\sigma \overrightarrow{\mathrm{E}}),
$$

with $\overrightarrow{\mathrm{E}}$ being the electric field. The equation of state (4) is obtained ${ }^{5}$ from the equality of the second-order mixed derivatives of (2). From (2) and (3) it may be seen that a nonvanishing relaxation time $\tau$ in (5) has some repercussions in the entropy and in the temperature, so that it is not coherent to retain the usual local-equilibrium entropy if one uses the generalized Ohm's law (5). Starting from (2), for the second differential of the generalized entropy, one obtains

$$
\begin{aligned}
d^{2} s= & -\left[\left(c T^{2}\right)^{-1}+\frac{1}{2}\left(\frac{\partial^{2} \alpha}{\partial u^{2}}\right) \overrightarrow{\mathbf{J}}_{0}^{2}\right](d u)^{2} \\
& -\alpha d \overrightarrow{\mathbf{J}} \cdot d \overrightarrow{\mathbf{J}}-2\left(\frac{\partial \alpha}{\partial u}\right) \overrightarrow{\mathbf{J}}_{0} \cdot d \overrightarrow{\mathbf{J}} d u,
\end{aligned}
$$

where $c$ is the specific heat per unit mass and $\overrightarrow{\mathbf{J}}_{0}$ is the mean nonequilibrium value of $\overrightarrow{\mathbf{J}}$ given by $\overrightarrow{\mathrm{J}}_{0}=\sigma \overrightarrow{\mathrm{E}}$.

\section{NONEQUILIBRIUM FLUCTUATIONS OF THE ELECTRIC CURRENT}

As in the classical theory, we assume that the probability of the fluctuations at constant temperature and constant electric field is given by (see Ref. 12, Chap. 15)

$$
\begin{aligned}
W \sim \exp \left[( \frac { 1 } { k } ] \left\{S(\hat{u}, \hat{\vec{J}})-\Theta_{0}^{-1} \hat{u}+\left[\frac{\tau v}{\sigma T}\right] \overrightarrow{\mathbf{J}}_{0} \cdot \hat{\overrightarrow{\mathbf{J}}}\right.\right. \\
\left.\left.-S\left[\Theta_{0}^{-1},-\left[\frac{\tau v}{\sigma T}\right] \overrightarrow{\mathbf{J}}_{0}\right]\right\}\right],
\end{aligned}
$$

where $\hat{u}$ and $\hat{\vec{J}}$ are the instantaneous values of $u$ and $\overrightarrow{\mathrm{J}}$, while $\Theta_{0}^{-1}$ and $(\tau v / \sigma T) \overrightarrow{\mathbf{J}}_{0}=(\tau v / T) \overrightarrow{\mathrm{E}}$, are the fixed values of the corresponding parameters $\Theta^{-1}=\partial s / \partial u$ and $(\tau v / \sigma T) \overrightarrow{\mathbf{J}}=-\partial s / \partial \overrightarrow{\mathbf{J}}$. On the other hand, $S\left[\Theta_{0}^{-1},-(\tau v / \sigma T) \overrightarrow{\mathbf{J}}_{0}\right]$ is the corresponding Legendre transform of $S$, given by

$$
\begin{aligned}
S\left[\Theta_{0}^{-1},-\left[\frac{\tau v}{\sigma T}\right] \overrightarrow{\mathrm{J}}_{0}\right]= & S-\Theta_{0}^{-1} u \\
& +\left(\frac{\tau v}{\sigma T}\right) \overrightarrow{\mathrm{J}}_{0} \cdot \overrightarrow{\mathrm{J}} .
\end{aligned}
$$

Expanding $s(\hat{u}, \hat{\vec{J}})$ around the mean value $s\left(u_{0}, \overrightarrow{\mathrm{J}}_{0}\right)$ in powers of the deviations $\delta u, \delta \overrightarrow{\mathbf{J}}$, and neglecting higher-order terms, it is easily found that (7) may be approximated by (Ref. 12, Chap. 15)

$$
W \sim \exp \left(\frac{\delta^{2} s}{2 k}\right),
$$

which predicts the second moments correctly although it is not accurate in the prediction of the higher-order moments. We have shown ${ }^{6}$ that in equilibrium, (9) leads in a direct way to the classical Nyquist expression for the current fluctuations. The use of (9) with the local-equilibrium entropy has been criticized by some authors ${ }^{13}$ who have used an expression similar to (9) as a basis for the construction of nonequilibrium thermodynamic potentials from the knowledge of the fluctuations obtained, for instance, from a master equation. Here, in order to establish a connection with these attempts to extend classical thermodynamics to nonequilibrium steady states, we study the consequences of hypothesis (9) with the generalized entropy (2), which contains the dissipative fluxes and can therefore account for some nonequilibrium features.

From (6) and (9) we obtain for the second moments of the fluctuations the following expressions: 


$$
\begin{aligned}
& \langle\delta u \delta u\rangle=k c T^{2}\left(1+\Lambda \overrightarrow{\mathrm{J}}_{0}^{2}\right)^{-1}, \\
& \left\langle\delta u \delta J_{i}\right\rangle=-\left(\frac{k c T^{2}}{\alpha}\right)\left(\frac{\partial \alpha}{\partial u}\right) J_{0 i}\left(1+\Lambda \overrightarrow{\mathrm{J}}_{0}^{2}\right)^{-1}, \\
& \left\langle\delta J_{i} \delta J_{j}\right\rangle=\left(\frac{k c T}{\tau v}\right)\left(1+\Lambda \overrightarrow{\mathrm{J}}_{0}^{2}\right)^{-1}\left[\delta_{i j}+\left(\frac{c T^{2}}{2}\right)\left(\frac{\partial^{2} \alpha}{\partial u^{2}}\right) J_{0 i} J_{0 j}\right],
\end{aligned}
$$

with

$$
\begin{aligned}
\Lambda= & \left(\frac{c T^{2}}{2}\right)\left(\frac{\partial^{2} \alpha}{\partial u^{2}}\right) \\
& -\left(\frac{c T^{2}}{\alpha}\right)\left(\frac{\partial \alpha}{\partial u}\right)^{2} .
\end{aligned}
$$

Here, we are mainly interested in the currentcurrent fluctuations. Assuming that the applied electric field is low, we can develop $\left(1+\Lambda \overrightarrow{\mathrm{J}}_{0}^{2}\right)^{-1}$ in (12) obtaining for the one time correlation

$$
\langle\delta J(0) \delta J(0)\rangle=\left(\frac{k \sigma T}{\tau v}\right)\left[1+\left(c T^{2} / \alpha\right)\left[\frac{\partial \alpha}{\partial u}\right)^{2} \overrightarrow{\mathbf{J}}_{0}^{2}\right] \text {. }
$$

Now, taking into account the equation of state (3) and the well-known relation ${ }^{14} \sigma=\left(n e^{2} / m\right) \tau$ (with $n, e$, and $m$ the electron density, the electron charge, and the electron mass, respectively) we get

$$
\langle\delta J(0) \delta J(0)\rangle=\left(\frac{k \sigma T}{\tau v}\right)\left[1+\left(\frac{n e^{2} T}{m c}\right) v\left(\frac{E \tau}{T}\right)^{2}\right] .
$$

It can be shown ${ }^{11}$ that the relaxation time appearing in the electrical conductivity is indeed the same as that appearing in (5). This relaxation time $\tau$ is related to the mean-free path $l$ by $\tau=l v_{F}^{-1}$ $=\operatorname{lm}\left(k_{F} \hbar\right)^{-1}$ with $k_{F}$ being the Fermi wave vector of the system. Keeping in mind that the specific heat per unit mass for the electron gas is given by $^{14}$

$$
c=\left(\pi^{2} / 2\right)\left(k^{2} T / \epsilon_{F} m\right),
$$

with $\epsilon_{F}$ the Fermi energy given by $\epsilon_{F}=\left(\hbar k_{F}\right)^{2} / 2 m$, we obtain from (14),

$$
\langle\delta J(0) \delta J(0)\rangle=\left(\frac{k \sigma T}{\tau v}\right)\left[1+\pi^{-2}\left(\frac{e E l}{k T}\right)^{2}\right] .
$$

In terms of the total intensity $I=A J$, in a resistor of cross section $A$ and length $L$, and taking into account the evolution for the fluctuations (5), we get for the spectral distribution of the timecorrelation function

$$
\begin{aligned}
\langle\delta I \delta I\rangle_{\omega}= & \left(\frac{2 k T}{R}\right)\left[1+\pi^{-2}\left(\frac{e E l}{k T}\right)^{2}\right] \\
& \times\left(1+\omega^{2} \tau^{2}\right)^{-1},
\end{aligned}
$$

where $R=L / \sigma A$ is the electrical resistance. For low frequencies such that $\omega \tau<<1,(16)$ reduces to

$$
\langle\delta I \delta I\rangle_{\omega}=\left(\frac{2 k T}{R}\right)\left[1+\pi^{-2}\left(\frac{e E l}{k T}\right)^{2}\right] .
$$

This expression gives the second moments of the fluctuations of the electric current near the constant value $\overrightarrow{\mathrm{J}}_{0}=\sigma \overrightarrow{\mathrm{E}}$. However, due to the Joule heating, the system is not a stationary state, and the temperature gradually rises, according to (1). Since the dominant term in (17) is proportional to $T$ it will diverge at long times. In order to eliminate this divergence, one would have to cool the system. It can be easily shown that the inclusion of a heat flux perpendicular to the electric current leaves (17) unchanged, so that in a true steady state (when the heat generated by the electric field and the thermal energy flux produced by a thermal gradient balance each other) the electric current fluctuations are also given by (17) with a constant $T$.

We may compare our phenomenological result (17) with the autocorrelation function obtained by Tremblay et $a l .{ }^{9}$ by a cumbersome nonequilibrium diagrammatic perturbation theory. They assume a system of metallic electrons that interact only with dilute, static, and isotropically scattering impurities. Their result is, after removing some divergences arising from the Joule heating,

$$
\langle\delta I \delta I\rangle_{\omega}=\left[\frac{2 k T}{R}\right]\left[1+0.156\left(\frac{e E l}{k T}\right)^{2}\right] .
$$

Recently, Tremblay and Vidal ${ }^{10}$ have given a simpler derivation of the nonequilibrium correction 
in (18) by means of kinetic arguments. For the leading term, due to the second-order contribution $f^{(2)}$ to the stationary distribution function, they found a value $0.100(e E l / k T)^{2}$, while for the contribution arising from electron collisions, due to terms of the form $f^{(1)} f^{(1)}$, with $f^{(1)}$ the first-order term in the stationary distribution function, they calculate $0.056(e E l / k T)^{2}$. As it may be seen, our result is in good agreement with the leading corrections of the microscopic calculations, but it does not give the collision contribution. In fact, as pointed out by Gantsevich et al. ${ }^{15}$ and Lax, ${ }^{1}$ and as commented on by Tremblay and Vidal, ${ }^{10}$ it may be seen from detailed microscopic calculations that entropy considerations are accurate only in the case when the stationary distribution function satisfies detailed balance, or when Boltzmann statistics are obeyed. Since in the present problem these conditions are not fulfilled, the disagreement between both results is not surprising.

A different problem is that in nonequilibrium the temperature of the metallic resistor, due to Joule heating, is not the same as its equilibrium temperature. In principle, Eq. (17) should be applied with the local value of the temperature determined by the Joule heating and the loss mechanism.

\section{CONCLUSIONS}

Our aim in this paper was to explore the applicability of a phenomenological generalized entropy to nonequilibrium fluctuations, in order to obtain a deeper insight into the signification and the limits of a nonequilibrium entropy. The method outlined in this paper cannot account for corrections due to particle-particle collisions. As we have pointed out, this limitation was already known from microscopic considerations for statistical entropies. ${ }^{1,10,15}$ In spite of this shortcoming, its results for corrections due to relaxational effects are in very good agreement with the values obtained from microscopic methods.

It must be noted that, in contrast with previous work on this subject, no microscopic considerations have entered into the definition of our nonequilibrium entropy, which has been obtained from purely macroscopic methods. This gives to the present procedure its particular simplicity. Since it has been obtained by comparison with relaxational constitutive equations for the dissipative fluxes, a possible way to improve the present results could be a comparison with more elaborate models for the evolution equations for the dissipative fluxes. This would lead us too far away from our present purpose: to recover from a phenomenological entropy some results of nonequilibrium fluctuations which are clearly beyond the scope of classical irreversible thermodynamics.

\section{ACKNOWLEDGMENTS}

This work has been partially supported by the Comisión Asesora de Investigación Cientifica y Técnica of the Spanish Government.
${ }^{1}$ M. Lax, Rev. Mod. Phys. 32, 25 (1960).

${ }^{2}$ Stochastic Processes in Nonequilibrium Systems, edited by L. Garrido, P. Seglar, and P. Shepperd, Sitges International School of Statistical Mechanics (Springer, Berlin, 1978).

${ }^{3}$ J. Keizer, J. Chem. Phys. 69, 2609 (1978); I. Procaccia, D. Ronis, and I. Oppenheim, Phys. Rev. A 20, 2533 (1979); D. Ronis, I. Procaccia, and J. Machta, ibid. 22, 714 (1980); A.-M.S. Tremblay, M. R. Arai, and E. Siggia, ibid. 23, 1451 (1981).

${ }^{4}$ G. Lebon, D. Jou, and J. Casas-Vázquez, J. Phys. A $\underline{13}, 275$ (1980).

5J. Casas-Vázquez and D. Jou, J. Phys. A $\underline{14}, 1225$ (1981).

${ }^{6}$ D. Jou and J. E. Llebot, J. Phys. A 13 , L47 (1980).

${ }^{7}$ D. Jou and J. Casas-Vázquez, J. Non-Equilib. Thermodyn. $\underline{5}, 91$ (1980).
${ }^{8}$ D. Jou, J. E. Llebot, and J. Casas-Vázquez, Phys. Rev. A 25, 508 (1982).

${ }^{9}$ A.-M. S. Tremblay, B. Patton, P. C. Martin, and P. F. Maldague, Phys. Rev. A 19, 1721 (1979).

${ }^{10}$ A.-M. S. Tremblay and F. Vidal, report (unpublished).

11P. Glansdorff and I. Prigogine, Structure, Stability and Fluctuations (Wiley, New York, 1971).

${ }^{12}$ H. B. Callen, Thermodynamics (Wiley, New York, 1960).

13J. Keizer, in Pattern Formation by Dynamic Systems and Pattern Recognition, edited by H. Haken (Springer, Berlin, 1979), p. 266.

${ }^{14}$ G. H. Wannier, Statistical Physics (Wiley, New York, 1966).

${ }^{15}$ S. V. Gantsevich, V. L. Gurevich, and R. Katilius, Riv. Nuovo Cimento $\underline{2}, 1$ (1979). 